ISSN: 2224-0616

Int. J . Agril. Res. Innov. \& Tech. 5(1): 15-21, June, 2015

Available online at http:// www.ijarit.webs.com

\title{
GROW OUT TEST AND DETERMINATION OF PHYSICAL CHARACTERISTICS OF SEED OF RICE LANDRACES
}

\author{
M.S. Alam ${ }^{*}$, M.M. Islam² and S.N. Begum ${ }^{3}$ \\ Received 23 March 2015, Revised 26 April 2015, Accepted 26 June 2015, Published online 30 June 2015
}

\begin{abstract}
The study was attempted with an objective to check the purity of commonly used farmer's rice seed stock. Assessment of varietal purity of collected 76 rice genotypes showed that there was a variation among the genotypes for varietal purity. Thirty-two genotypes showed $100 \%$ varietal purity and forty-four genotypes were contaminated with different types of offtypes. The lowest varietal purity was observed in Lal gotal (74.67\%). Grain dimension and 1000 seed weight were measured as important physical characteristics of seed to identify varietal purity.
\end{abstract}

Keywords: Length-width Ratio, Stable Varietal Property, Homozygous Plant, Isolation Distance

\footnotetext{
${ }^{1}$ Department of Genetics and Plant Breeding, Bangladesh Agricultural University, Mymensingh, Bangladesh

${ }^{2}$ Principal Scientific Officer, Biotechnology Division, Bangladesh Institute of Nuclear Agriculture, Mymensingh, Bangladesh

${ }^{3}$ Senior Scientific Officer, Plant Breeding Division, Bangladesh Institute of Nuclear Agriculture, Mymensingh, Bangladesh

*Corresponding author's email: agt.shahed@gmail.com (M.S. Alam)
}

\section{Introduction}

A large number of the farmers of Bangladesh are adopted with local landraces and they are cultivating these for long period with traditional cultural practices. As a result, the yield of those varieties is decreasing due to genetic impurities through mechanical mixtures, incorporating with off-types or volunteer plants, natural outcrossing, mutations etc. Samples of cultivars from farmer's fields are rarely homogenous in genetic composition. They contain varying degrees of differences in morphological or physiological aspects, some obvious to the eye while some others are not. Generally, a farmer's variety can be differentiated into two or more subpopulations that are distinct in one or more recognizable traits (Chang, 1976). Landraces are generally low yielding, but have high yield stability and resistance against many biotic and abiotic stresses. Conventionally grow out test (GOT) is used to assay the varietal purity of rice seed lots on a representative sample of the seeds (Moorthy et al., 2011, Naresh et al., 2009, Sundaram et al., 2008, Sang et al., 2006). In GOT, plants are grown up to maturity to identify the off-types through assessing several morphological and floral characteristics that distinguish the genotypes (Yashitola et al., 2002). GOT is time consuming (takes one full growing season for completion), space demanding and often does not allow the unequivocal identification of genotypes. The molecular markers are of great importance for rapid assessment seed purity (Yashitola et al., 2002,
Antonova et al., 2006 and Pallavi et al., 2011). Rajendrakumar et al. (2007) used molecular markers for detection of contaminant in CMS seed stocks of rice and confirm result based on observation recorded in GOT field. Therefore, this experiment was conducted to evaluate the varietal purity through GOT and important seed physical characters were determined according to IRRI guidelines.

\section{Materials and Methods}

The experiment was conducted at the experimental farm of Bangladesh Institute of Nuclear Agriculture (BINA), Mymensingh, during July 2011 to December 2012. Geographically the experimental area is located at $24075 \mathrm{~N}$ latitude and $9005 \mathrm{E}$ longitudes at the elevation of $18 \mathrm{~m}$ above the sea level. The field was medium high land. The soil of the experimental site belonging under the agro-ecological zone of Old Brahmaputra Floodplain (AEZ-9). The experimental field area was under sub-tropical climates characterized by heavy rainfall during the month of April to September and scanty rainfall during October to March. Fifty four rice landraces (i.e. Dudh kalam, Hati bajore, Malagoti, Kuchra, Enghi, Kajol shail, Hogla, Jamai naru, Hari, Dakh shail, Moina moti, Marish shail, Patnai, Bhute shallot, Kute patnai, Mohini shallot, Moghai balam, Sada gotal, Khak shail, Mohime, Holde gotal, J ota balam, Tilek kuchi, Rani Shalot, Kathi goccha, Bashful balam, 
Bazra muri, Durga bhog, Kumra ghor, Khainol, Ghunshi, Chinikani, Dhar shail, Khejur chori, Shaheb kachi, Raja shail, Hamai, Mura bajal, Lal gotal, Kalmilata, Volanath, Rupessor, Sylhet balam, Karengal, Kalo mota, Mota aman, Ghochi, Chap shail, Mondeshor, Nona kochi, Ghocca, Tal mugur, Ghigoj and Tor balam) were collected from the farmer's field of southern Bangladesh. Rest of the rice genotypes (21 landraces i.e. Fulkainja, Piarjat, Koicha binni, Lal biroi, Lalanamia, Golapi, Asam binni, Kakua binni, Nona bokhra, J ongli boro, Kashrail, Ledra binni, Nunnia, Rotisail, Genggeng binni, Chinisail, Jolkumri, Ponkhiraj, Mowbinni, Bogi, Kali boro and Binadhan-8) was collected from Plant Breeding Division, BINA, Mymensingh. The experiment was laid out in a Randomized Complete Block Design (RCBD) with three replications. The experimental field was divided into three blocks each representing one replication. Each block was then sub-divided in to seventy-six plots. The seventy-six rice genotypes were placed in each plot. The size of the unit plot was one square meter in size. Row to row and plant to plant distances were $20 \mathrm{~cm}$ and $20 \mathrm{~cm}$, respectively in each plot.

\section{Grow out test (GOT)}

Data were recorded on off-types from per plot in each block, which were not identified at earlier during seed processing after collection. Among the studied characters, number of off-types meter $^{2}$ and types of off-types meter ${ }^{2}$ were recorded in the field and the remaining two characters were calculated and recorded in the laboratory, which were as follows:

\section{Number of off-types meter-2}

By regular field visits, off-types were identified in each plots and marking of off-types with tag was done on a regular basis. Panicles of the off-types were covered with bags made of glossy papers to avoid further out crossing in order to maintain genetic purity of the rice genotypes of the plot. Selection criteria for off-types at different stages of crops followed in this experiment is shown in Table 1. The land was free from volunteer plants.

Table 1. Off-type selection criteria followed in the experiment

\begin{tabular}{ll}
\hline Stage of crop & Selection criteria \\
\hline Maximum tillering & Very tall or short plants \\
Flowering stage & Early \& late flowering plants \\
Pre-harvest stage & Awned grains \\
Harvest stage & Seed size, shape and color \\
\hline
\end{tabular}

\section{Ty pes of off-types meter-2}

At harvesting stage, off-types were classified into different types based on the plant height, difference in seed size, shape, presence or absence of awns and seed coat color. Similarities of these characters among the off-types were considered as same off-type genotypes in this experiment.

Percent $(\%)$ off-types meter ${ }^{2}=($ Number of off-types meter ${ }^{2 / N u m b e r}$ of total plant meter $\left.{ }^{-2}\right) \times 100$

In this experiment, Number of total plant meter ${ }^{2}$ was 25 in each case.

Percent (\%) homozygous plant meter $^{2}=100$ - Percent (\%) off-types meter ${ }^{2}$

\section{Measurement of physical characteristics rice seed}

Grain dimensions (length-width ratio) were measured according to the standard rice grain size and shape scale followed in IRRI and is shown in Tables $2 \& 3$.

Table 2. Size classification of rice grains

\begin{tabular}{lll}
\hline Scale & Size category & Length \\
\hline 1 & Extra long & $>7.5 \mathrm{~mm}$ \\
3 & Long & 6.61 to $7.5 \mathrm{~mm}$ \\
5 & Medium & 5.51 to $6.6 \mathrm{~mm}$ \\
7 & Short & $\leq 5.5 \mathrm{~mm}$ \\
\hline
\end{tabular}

(Source: www.irri.org)

Table 3. Shape classification of rice grains

\begin{tabular}{lll}
\hline Scale & Shape & Ratio \\
\hline 1 & Slender & Over 3.0 \\
3 & Medium & 2.1 to 3.0 \\
5 & Bold & 1.1 to 2.0 \\
9 & Round & Less than 1.1 \\
\hline
\end{tabular}

(Source: www.irri.org)

One thousand clean sun dried grains were counted from the sample plant by seed counter after which weighed (g) was taken at $14 \%$ seed moisture content (with husk). After first round of the purification, a second round of purification was done and seeds are preserved at the germplasm of Plant Breeding Division, BINA, Mymensingh. Analysis of variance was performed using the plant breeding statistical program (PLBSTAT, Version 2N, Utz 2007).

\section{Results and Discussion}

\section{Grow out test (GOT)}

The analysis of variance for evaluation of varietal purity of 76 rice landraces is shown in Table 4. Analysis of variance showed that there were significant variations among the genotypes for all the characters studied. This indicates that there was a variation among the genotypes for varietal purity. 
Table 4. Analysis of variance for evaluation of varietal purity of 76 rice genotypes

\begin{tabular}{cccccc}
\hline Items & d.f. & $\begin{array}{c}\text { Number of off- } \\
\text { types meter }\end{array}$ & $\begin{array}{c}\text { Types of off- } \\
\text { types meter }\end{array}$ & $\begin{array}{c}\text { Percent (\%) off- } \\
\text { types meter }\end{array}$ & $\begin{array}{c}\text { Percent (\%) } \\
\text { homozygous plant } \\
\text { meter }^{2}\end{array}$ \\
\hline Genotypes & 75 & $23.27^{* *}$ & $23.74^{* *}$ & $23.27^{* *}$ & $23.27^{* *}$ \\
Replication & 2 & $11.28^{* *}$ & $18.70^{* *}$ & $11.28^{* *}$ & $11.28^{* *}$ \\
Error & 150 & 0.4211 & 0.2561 & 6.7378 & 6.7378 \\
\hline
\end{tabular}

** indicates significant at 0.01 probability

In Table 5 it was found that number of off-types meter $^{2}$ ranged from 0 to 6.33. Out of 76 genotypes, the highest number of off-type was observed in Lal gotal, which was closely followed, by J ota balam, Mura bajal and Mohime. Thirtytwo genotypes were free from off-types. The range of types of off-types meter ${ }^{2}$ was 0 to 5 and the mean value for this trait is 1.20 . The highest types of off-types were recorded in the plot of Lal gotal followed by Mura bajal, Jota balam and Mohime.

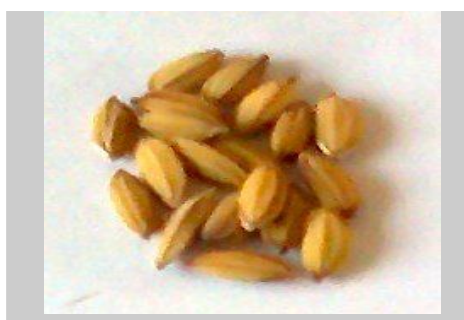

Main Variety

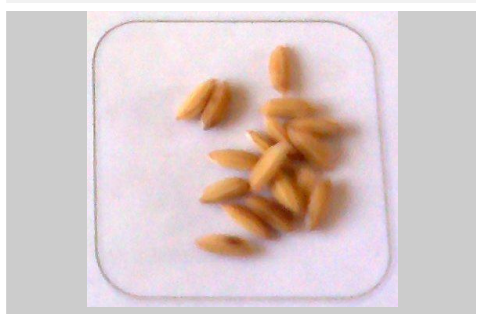

Off-type 03

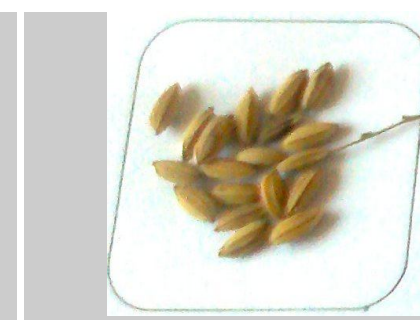

Off-type 01

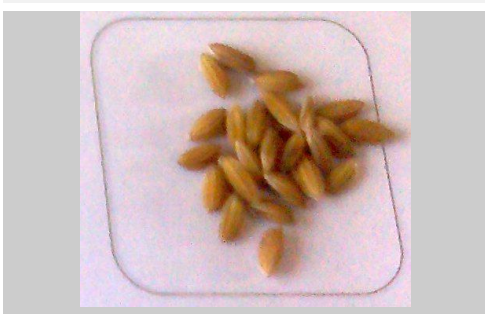

Off-type 04

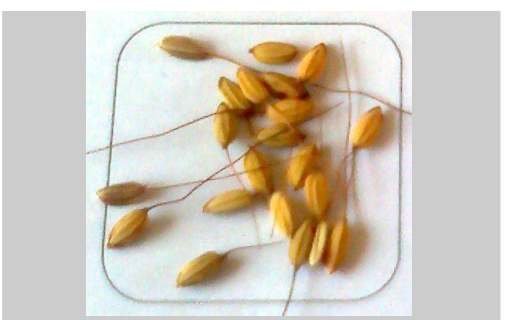

Off-type 02

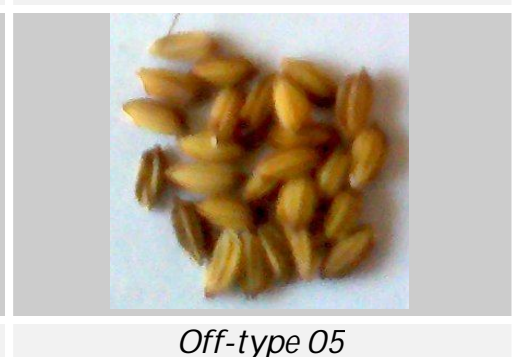

Off-type 05

\section{Genotype: Mohime}

Fig. 1. Different types of off-types found during varietal purification of the landrace Mohime

The highest percent (\%) of off-types was recorded in Lal gotal (25.33\%) followed by Jota balam (24\%), Mura bajal (24\%) and Mohime (21.33\%). The range for this trait was 0 to 25.33. In this experiment, thirty-two genotypes showed 100\% varietal purity and forty-four genotypes were contaminated with different types of off-types. The lowest varietal purity was observed in Lal gotal (74.67\%). This indicates that a majority percent of landraces are in a process of varietal deterioration. 
Table 5. Mean performance of 76 rice genotypes for evaluation of varietal purity

\begin{tabular}{|c|c|c|c|c|}
\hline Genotypes & $\begin{array}{c}\text { Number of off-types } \\
\text { meter }^{2}\end{array}$ & $\begin{array}{c}\text { Types of off-types } \\
\text { meter }^{2}\end{array}$ & $\begin{array}{l}\text { Percent (\%) off- } \\
\text { types meter-2 }\end{array}$ & $\begin{array}{l}\text { Percent (\%) homozygous } \\
\text { plant meter }{ }^{2}\end{array}$ \\
\hline Dudh kalam & 2.33 & 1.00 & 9.33 & 90.67 \\
\hline Hati bajore & 2.67 & 1.33 & 10.67 & 89.33 \\
\hline Malagoti & 0.00 & 0.00 & 0.00 & 100.00 \\
\hline Kuchra & 2.67 & 2.67 & 10.67 & 89.33 \\
\hline Enghi & 0.00 & 0.00 & 0.00 & 100.00 \\
\hline Kajol shail & 0.00 & 0.00 & 0.00 & 100.00 \\
\hline Hogla & 3.67 & 2.00 & 14.67 & 85.33 \\
\hline J amai naru & 0.00 & 0.00 & 0.00 & 100.00 \\
\hline Hari & 0.00 & 0.00 & 0.00 & 100.00 \\
\hline Dakh shail & 0.00 & 0.00 & 0.00 & 100.00 \\
\hline Moina moti & 1.00 & 0.67 & 4.00 & 96.00 \\
\hline Marish shail & 3.33 & 2.67 & 13.33 & 86.67 \\
\hline Patnai & 2.33 & 1.67 & 9.33 & 90.67 \\
\hline Bhute shalot & 2.33 & 2.00 & 9.33 & 90.67 \\
\hline Kute patnai & 0.67 & 0.67 & 2.67 & 97.33 \\
\hline Mohini shalot & 4.67 & 2.00 & 18.67 & 81.33 \\
\hline Moghai balam & 0.00 & 0.00 & 0.00 & 100.00 \\
\hline Sada gotal & 4.00 & 3.67 & 16.00 & 84.00 \\
\hline Khak shail & 1.67 & 1.00 & 6.67 & 93.33 \\
\hline Mohime & 5.33 & 4.33 & 21.33 & 78.67 \\
\hline Holde gotal & 0.00 & 0.00 & 0.00 & 100.00 \\
\hline J ota balam & 6.00 & 4.33 & 24.00 & 76.00 \\
\hline Tilek kuchi & 4.33 & 4.00 & 17.33 & 82.67 \\
\hline Rani shalot & 4.67 & 3.67 & 18.67 & 81.33 \\
\hline Kathi goccha & 0.00 & 0.00 & 0.00 & 100.00 \\
\hline Bashful balam & 0.00 & 0.00 & 0.00 & 100.00 \\
\hline Bazra muri & 3.00 & 2.67 & 12.00 & 88.00 \\
\hline Durga bhog & 2.67 & 2.33 & 10.67 & 89.33 \\
\hline Kumra ghor & 3.67 & 3.33 & 14.67 & 85.33 \\
\hline Khainol & 4.33 & 4.00 & 17.33 & 82.67 \\
\hline Ghunshi & 3.33 & 1.00 & 13.33 & 86.67 \\
\hline Chinikani & 0.00 & 0.00 & 0.00 & 100.00 \\
\hline Dhar shail & 4.00 & 1.00 & 16.00 & 84.00 \\
\hline Khejur chori & 2.67 & 2.67 & 10.67 & 89.33 \\
\hline Shaheb kachi & 0.33 & 0.33 & 1.33 & 98.67 \\
\hline Raja shail & 4.00 & 3.67 & 16.00 & 84.00 \\
\hline Hamai & 0.00 & 0.00 & 0.00 & 100.00 \\
\hline Mura bajal & 6.00 & 4.67 & 24.00 & 76.00 \\
\hline Lal gotal & 6.33 & 5.00 & 25.33 & 74.67 \\
\hline Kalmilata & 0.33 & 0.33 & 1.33 & 98.67 \\
\hline Volanath & 1.33 & 1.33 & 5.33 & 94.67 \\
\hline Rupessor & 2.33 & 1.00 & 9.33 & 90.67 \\
\hline Sylhet balam & 0.00 & 0.00 & 0.00 & 100.00 \\
\hline Karengal & 2.33 & 2.33 & 9.33 & 90.67 \\
\hline Kalo mota & 0.67 & 0.67 & 2.67 & 97.33 \\
\hline Mota aman & 1.33 & 1.33 & 5.33 & 94.67 \\
\hline Ghochi & 1.67 & 1.67 & 6.67 & 93.33 \\
\hline Chap shail & 2.67 & 2.00 & 10.67 & 89.33 \\
\hline Mondeshor & 3.00 & 2.67 & 12.00 & 88.00 \\
\hline Nona kochi & 2.00 & 1.67 & 8.00 & 92.00 \\
\hline Ghocca & 1.67 & 1.33 & 6.67 & 93.33 \\
\hline Tal mugur & 0.00 & 0.00 & 0.00 & 100.00 \\
\hline Ghigoj & 0.00 & 0.00 & 0.00 & 100.00 \\
\hline Tor balam & 1.67 & 1.00 & 6.67 & 93.33 \\
\hline Fulkainja & 0.00 & 0.00 & 0.00 & 100.00 \\
\hline Piarjat & 0.00 & 0.00 & 0.00 & 100.00 \\
\hline Koicha binni & 0.00 & 0.00 & 0.00 & 100.00 \\
\hline Lal biroi & 0.33 & 0.33 & 1.33 & 98.67 \\
\hline Lalanamia & 0.00 & 0.00 & 0.00 & 100.00 \\
\hline Golapi & 0.00 & 0.00 & 0.00 & 100.00 \\
\hline Asam binni & 0.00 & 0.00 & 0.00 & 100.00 \\
\hline Kakua binni & 0.00 & 0.00 & 0.00 & 100.00 \\
\hline Nona bokhra & 0.67 & 0.67 & 2.67 & 97.33 \\
\hline J ongli boro & 2.33 & 2.00 & 9.33 & 90.67 \\
\hline Kashrail & 0.00 & 0.00 & 0.00 & 100.00 \\
\hline Ledra binni & 0.00 & 0.00 & 0.00 & 100.00 \\
\hline Nunnia & 0.00 & 0.00 & 0.00 & 100.00 \\
\hline Rotisail & 2.67 & 1.67 & 10.67 & 89.33 \\
\hline Gengeng binni & 0.00 & 0.00 & 0.00 & 100.00 \\
\hline Chinisail & 0.00 & 0.00 & 0.00 & 100.00 \\
\hline J olkumri & 0.00 & 0.00 & 0.00 & 100.00 \\
\hline Ponkhiraj & 0.00 & 0.00 & 0.00 & 100.00 \\
\hline Mowbinni & 0.00 & 0.00 & 0.00 & 100.00 \\
\hline Bogi & 0.00 & 0.00 & 0.00 & 100.00 \\
\hline Kali boro & 1.00 & 0.67 & 4.00 & 96.00 \\
\hline Binadhan- 8 & 0.00 & 0.00 & 0.00 & 100.00 \\
\hline CV (\%) & 41.10 & 42.27 & 41.10 & 2.77 \\
\hline Maximum & 6.33 & 5 & 25.33 & 100 \\
\hline Minimum & 0 & 0 & 0 & 74.67 \\
\hline Mean & 1.58 & 1.20 & 6.32 & 93.68 \\
\hline LSD (0.05) & 1.05 & 0.82 & 4.19 & 4.19 \\
\hline
\end{tabular}




\section{Measurement of physical characteristics of rice seed}

In case of grain size, seven genotypes were categorized as long; fifty-five as medium and fourteenth as short grain (Table 6). The range for this trait was 4.26 to 7.19 . The highest grain length was observed in Rupessor and Asam binni (7.19 mm). Chinishail exhibited the lowest grain size $(4.26 \mathrm{~mm})$ (Table 8$)$.

Table 6. Size classification of 76 rice grains

\begin{tabular}{lcc}
\hline Genotypes & Size category & Length \\
\hline $\begin{array}{l}\text { Hati bajore, Moghai balam, Bashful balam, Kalmilata, } \\
\text { Rupessor, Tal mugur, Asam binni }\end{array}$ & Long & 6.61 to $7.5 \mathrm{~mm}$ \\
\hline $\begin{array}{l}\text { Dudh kalam, Malagoti, Kuchra, Kajol shail, Hogla, Jamai } \\
\text { naru, Marish shail, Patnai, Bhute shalot, Kute patnai, }\end{array}$ & \\
$\begin{array}{l}\text { Mohini shalot, Sada gotal, Khak shail, Mohime, Holde gotal, } \\
\text { Jota balam, Tilek kuchi, Rani shalot, Kathi goccha, Durga } \\
\text { bhog, Kumra ghor, Khainol, Khejur chori, Shaheb kachi, }\end{array}$ & \\
$\begin{array}{l}\text { Raja shail, Hamai, Mura bajal, Lal gotal, Volanath, Sylhet } \\
\text { balam, Karengal, Kalo mota, Mota aman, Chap shail, }\end{array}$ & Medium & \\
$\begin{array}{l}\text { Mondeshor, Nona kochi, Ghocca, Ghigoj, Tor balam, } \\
\text { Fulkainja, Piarjat, Koicha binni, Lal biroi, Lalanamia, Nona } \\
\text { bokhra, Kashrail, Ledra binni, Nunnia, Rotisail, Genggeng }\end{array}$ & & \\
binni, Ponkhiraj, Mowbinni, Bogi, Kali Boro, Binadhan-8 & & \\
\hline $\begin{array}{l}\text { Enghi, Hari, Dakh shail, Moina moti, Bazra muri, Ghunshi, } \\
\text { Chinikani, Dhar shail,Ghochi, Golapi, Kakua binni, Jongli } \\
\text { boro, Chinishail, Jolkumri }\end{array}$ & Short & \\
\hline
\end{tabular}

With respect to shape, eight rice grains were evaluated as slender as the grain length width ratio was more than 3.0; fifty seven rice grains were considered as medium as the values were

Table 7. Shape classification of 76 rice grains existed between 2.1 and 3.0; and eleven rice grains were estimated as bold as GLWR values were existed between 1.1 and 2.0 (Table 7).

\begin{tabular}{lcc}
\hline Genotypes & Shape & Ratio \\
\hline Malagoti, Moghai balam, Bashful balam, Rupessor, Fulkainja, & Slender & $>3.0$ \\
Koicha binni, Asam binni, Nunnia & & \\
\hline Dudh kalam, Hati bajore, Kajol shail, Hogla, Jamai naru, Hari, & \\
Marish shail, Patnai, Bhute shalot, Kute patnai, Mohini shalot, Sada & \\
gotal, Khak shail, Holde gotal, J ota balam, Tilek kuchi, Rani shalot, & \\
Kathi goccha, Durga bhog, Kumra ghor, Khainol, Chinikani, Khejur & \\
chori, Shaheb kachi, Raja shail, Hamai, Mura bajal, Lal gotal, & Medium & 2.1 to 3.0 \\
Kalmilata, Volanath, Kalo mota, Ghochi, Chap shail, Mondeshor, & \\
Nona kochi, Ghocca, Tal mugur, Ghigoj, Tor balam, Piarjat, Lal & \\
biroi, Lalanamia, Golapi, Kakua binni, Nona bokhra, J ongli boro, & \\
Kashrail, Ledra binni, Rotisail, Genggeng binni, Chinisail, Jolkumri, & \\
Ponkhiraj, Mowbinni, Bogi, Kali boro, Binadhan-8 & \\
\hline $\begin{array}{l}\text { Kuchra, Enghi, Dakh shail, Moina moti, Mohime, Bazra muri, } \\
\text { Ghunshi, Dhar shail, Sylhet balam, Karengal, Mota aman }\end{array}$ & \multirow{2}{*}{ Bold } \\
\hline
\end{tabular}

The range of 1000 -grain weight was $11.28 \mathrm{~g}$ to $31.93 \mathrm{~g}$. Highest 1000-grain was obtained from Mota aman $(31.93 \mathrm{~g})$ and the lowest was observed in Chinishail (11.28 g). The mean value for this trait is $24.23 \mathrm{~g}$. Seed physical characteristics of these genotypes are presented in Table 8 . In future, varietal purity of these genotypes could be determined through comparing with this data. 
Table 8. Physical characteristics of rice seed used to identify varietal purity

\begin{tabular}{|c|c|c|c|c|}
\hline Genotypes & Grain length (mm) & Grain width (mm) & $\begin{array}{c}\text { Grain length-width ratio } \\
\text { (GLWR) }\end{array}$ & 1000- grain weight (g) \\
\hline Dudh kalam & 6.10 & 2.70 & 2.26 & 28.15 \\
\hline Hati bajore & 6.90 & 2.90 & 2.38 & 31.23 \\
\hline Malagoti & 6.00 & 1.90 & 3.16 & 20.93 \\
\hline Kuchra & 5.80 & 2.90 & 2.00 & 28.67 \\
\hline Enghi & 4.70 & 2.80 & 1.68 & 20.59 \\
\hline Kajol shail & 6.20 & 2.55 & 2.43 & 29.27 \\
\hline Hogla & 6.10 & 2.90 & 2.10 & 27.84 \\
\hline Jamai naru & 5.92 & 2.80 & 2.11 & 25.52 \\
\hline Hari & 5.50 & 2.59 & 2.12 & 21.64 \\
\hline Dakh shail & 4.53 & 2.57 & 1.76 & 19.40 \\
\hline Moina moti & 4.80 & 2.60 & 1.85 & 21.60 \\
\hline Marish shail & 6.40 & 2.61 & 2.45 & 24.79 \\
\hline Patnai & 5.82 & 2.70 & 2.16 & 27.11 \\
\hline Bhute shalot & 6.02 & 2.80 & 2.15 & 28.14 \\
\hline Kute patnai & 6.27 & 2.47 & 2.54 & 27.40 \\
\hline Mohini shalot & 5.80 & 2.75 & 2.11 & 29.06 \\
\hline Moghai balam & 6.95 & 2.05 & 3.39 & 26.32 \\
\hline Sada gotal & 5.82 & 2.90 & 2.01 & 30.06 \\
\hline Khak shail & 6.29 & 2.65 & 2.37 & 28.61 \\
\hline Mohime & 5.80 & 2.95 & 1.97 & 28.44 \\
\hline Holde gotal & 6.15 & 2.59 & 2.37 & 26.80 \\
\hline Jota balam & 6.29 & 2.50 & 2.52 & 31.04 \\
\hline Tilek kuchi & 6.45 & 2.20 & 2.93 & 24.58 \\
\hline Rani shalot & 5.80 & 2.50 & 2.32 & 28.91 \\
\hline Kathi goccha & 5.59 & 2.50 & 2.24 & 25.09 \\
\hline Bashful balam & 6.79 & 2.01 & 3.38 & 23.76 \\
\hline Bazra muri & 4.90 & 2.80 & 1.75 & 21.31 \\
\hline Durga bhog & 5.82 & 2.40 & 2.43 & 21.12 \\
\hline Kumra ghor & 5.70 & 2.60 & 2.19 & 25.05 \\
\hline Khainol & 6.21 & 2.68 & 2.32 & 28.31 \\
\hline Ghunshi & 4.60 & 2.70 & 1.70 & 17.84 \\
\hline Chinikani & 4.30 & 2.06 & 2.09 & 12.35 \\
\hline Dhar shail & 4.35 & 2.50 & 1.74 & 14.13 \\
\hline Khejur chori & 6.02 & 2.70 & 2.23 & 27.49 \\
\hline Shaheb kachi & 5.68 & 2.77 & 2.05 & 26.27 \\
\hline Raja shail & 6.13 & 2.65 & 2.31 & 28.27 \\
\hline Hamai & 6.36 & 2.73 & 2.33 & 27.92 \\
\hline Mura bajal & 5.90 & 2.62 & 2.25 & 23.94 \\
\hline Lal gotal & 6.10 & 2.91 & 2.10 & 27.80 \\
\hline Kalmilata & 6.79 & 2.40 & 2.83 & 26.14 \\
\hline Volanath & 5.93 & 2.35 & 2.52 & 24.76 \\
\hline Rupessor & 7.19 & 2.05 & 3.51 & 25.44 \\
\hline Sylhet balam & 4.84 & 2.83 & 1.71 & 21.17 \\
\hline Karengal & 5.90 & 3.00 & 1.97 & 24.02 \\
\hline Kalo mota & 5.80 & 2.84 & 2.04 & 24.80 \\
\hline Mota aman & 5.70 & 3.00 & 1.90 & 31.93 \\
\hline Ghochi & 5.49 & 2.39 & 2.30 & 21.22 \\
\hline Chap shail & 6.43 & 2.80 & 2.30 & 28.72 \\
\hline Mondeshor & 6.30 & 2.80 & 2.25 & 29.50 \\
\hline Nona kochi & 6.10 & 2.90 & 2.10 & 28.50 \\
\hline Ghocca & 6.19 & 2.74 & 2.26 & 24.64 \\
\hline Tal mugur & 6.70 & 2.55 & 2.63 & 27.84 \\
\hline Ghigoj & 6.32 & 2.79 & 2.27 & 27.17 \\
\hline Tor balam & 6.50 & 2.58 & 2.52 & 27.65 \\
\hline Fulkainja & 6.00 & 1.99 & 3.02 & 17.14 \\
\hline Piarjat & 5.98 & 2.12 & 2.82 & 18.08 \\
\hline Koicha binni & 5.99 & 1.99 & 3.01 & 16.95 \\
\hline Lal biroi & 5.68 & 1.92 & 2.96 & 16.01 \\
\hline Lalanamia & 5.88 & 2.60 & 2.26 & 24.53 \\
\hline Golapi & 4.93 & 2.41 & 2.05 & 19.81 \\
\hline Asam binni & 7.19 & 2.12 & 3.39 & 24.51 \\
\hline Kakua binni & 4.72 & 1.78 & 2.65 & 11.75 \\
\hline Nona bokhra & 6.32 & 2.79 & 2.27 & 28.22 \\
\hline J ongli boro & 5.09 & 2.13 & 2.39 & 18.36 \\
\hline Kashrail & 6.50 & 2.60 & 2.50 & 27.36 \\
\hline Ledra binni & 5.91 & 2.29 & 2.58 & 21.66 \\
\hline Nunnia & 6.09 & 1.70 & 3.58 & 16.11 \\
\hline Rotisail & 6.24 & 2.50 & 2.50 & 23.40 \\
\hline Gengeng binni & 6.10 & 2.30 & 2.65 & 22.33 \\
\hline Chinisail & 4.26 & 1.75 & 2.43 & 11.28 \\
\hline Jolkumri & 5.48 & 2.59 & 2.12 & 22.71 \\
\hline Ponkhiraj & 5.91 & 2.20 & 2.69 & 22.57 \\
\hline Mowbinni & 5.80 & 2.67 & 2.17 & 25.18 \\
\hline Bogi & 5.70 & 2.70 & 2.11 & 25.34 \\
\hline Kali boro & 5.70 & 2.27 & 2.51 & 22.55 \\
\hline Binadhan- 8 & 5.89 & 2.60 & 2.27 & 25.51 \\
\hline Maximum & 7.19 & 3.00 & 3.58 & 31.93 \\
\hline Minimum & 4.26 & 1.70 & 1.68 & 11.28 \\
\hline Mean & 5.87 & 2.52 & 2.37 & 24.23 \\
\hline
\end{tabular}


Although we have now large number of HYV rice varieties, farmers do special affinity to landraces and substantial conservation of diverse landraces of rice in Bangladesh has continued since ancient times. The genuineness of the variety is one of the most important characteristics of good quality seed. A mixture of varieties in a field may significantly diminish the value of the crop due to poor yield, downgrading and reduce milling yield. Genetic purity test is done to verify any deviation from genuineness of the variety during its multiplications. The present study showed that conventional GOT test is an effective method of varietal purity test although morphological markers are time consuming and are environmental dependence.

\section{References}

Antonova, T.S., Guchetl, S.Z., Tchelustnikova, T.A. and Ramasanova, S.A. 2006. Development of marker system for identification and certification of rice lines and hybrids on the basis of SSR-analysis. Helia. 29: 63-72.

Chang, T.T. 1976. Manual on genetic conservation of rice germplasm for evaluation and utilization. International Rice Research Institute, Los Baños, Philippines. pp. 1-7.

Moorthy, K.K., Babu, P., Sreedhar, M., Sama VSAK, Kumar, P.N., Balachandran, S.M. and Sundaram, R.M. 2011 Identification of informative EST-SSR markers capable of distinguishing popular Indian rice varieties and their utilization in seed genetic purity assessments. Seed Sci \& Tech. 39(2):282292.

Naresh, V., Yamini, K.N., Rajendrakumar, P. and Kumar, V.D. 2009. EST-SSR marker-based assay for the genetic purity assessment of safflower hybrids. Euphytica 170(3): 347353.

Pallavi, H.M., Rame Gowda, Vishwanath, K., Shadakshari, Y.G. and Bhanuprakash, K. 2011. Identification of SSR markers for hybridity and seed genetic purity testing in sunflower (Helianthus annuus L.). Seed Sci. \&Tech. 39: 259-264.

Rajendrakumar, P., Biswal, A.K., Balachandran, S.M., Ramesha, M.S., Viraktamath, B.C. and Sundaram, R.M. 2007. A mitochondrial repeat specific marker for distinguishing wild abortive type cytoplasmic male sterile rice lines from their cognate isogenic maintainer lines. Crop Sci. 47: 207-211.

Sang, X., Yang, Z, Zhong, B., Li, Y., Hou, L., Pei, Y., Li, G. and He, G. 2006. Assessment of purity of rice CMS lines using cpDNA marker. Euphytica 152(2): 177-183.

Sundaram, R.M., Naveenkumar, B., Biradar, S.K., Balachandran, S.M., Mishra, B., Ilyas Ahmed, M., Viraktamath, B.C., Ramesha, M.S. and Sarma, N.P. 2008. Identification of informative SSR markers capable of distinguishing hybrid rice parental lines and their utilization in seed purity assessment. Euphytica 163(2): 215-224.

Utz, H.F. 2007. PLABSTAT (Version 2N), A computer program for the computation of variances and covariances. Institute of Plant Breeding, Seed Science, and Population Genetics, University of Hohenheim, Stuttgart, Germany (http:// www.unihohenheim. de/ipspwww/ soft.html cited 25 August 2007).

Yashitola, J., Thirumurugan, T., Sundaram, R.M., Naseerullah, M.K., Ramesha, M.S., Sarma N.P. and Sonti, R.V. 2002. Assessment of purity of rice hybrids using microsatellite and STS markers. Crop Sci. 42: 1369- 1373. 